\title{
$\mathbf{X}$.
}

\section{Zur Function des Corpus striatum. (Nucleus caudatus.)}

Experimentelle Studie.

Von Dr. Adolf Baginsky und Dr. Curt Lehmann.

(Aus dem physiologischen Laboratorium der Königl. landwirthschaftlichen Hochsehule in Berlin.)

Die Untersuchungen über die Function des Corpus striatum knüpfen an die von Magendie gemachten Angaben an. Magendie fand, dass Kaninchen, welchen beiderseits die Streifenhügel entfernt worden waren, von einem unwiderstehlichen Trieb zum Laufen ergriffen wurden. Einseitig operirte Thiere zeigten diesen Lauftrieb nicht, sondern wurden nur auffällig unruhig. Zum Theil wurden diese Angaben in der Folge völlig bestritten, zum Theil anders gedeutet, als von Magendie geschehen war. So bezogen Longet and Laffargue den auch von ihnen beobachteten Lauftrieb auf Schmerzensempfindungen oder auf Angstgefühl der Thiere, mit Fluchtversuchen u. s. w., die thatsächlichen Angaben wurden weiterhin von Schiff in mancher Richtung bestätigt und noch dahin ergänzt, dass die operirten Thiere, welchen gleichzeitig behufs Blosslegung der Streifenhügel die Grosshirnlappen abgetragen worden waren, erhebliche Störungen des Muskelgefühls zeigten. Schiff') leugnet indess jede specifische Irritation der Streifeuhügel und erkennt dieselben physiologisch nur als die Anfänge der Hirnlappen an, wobei er die beobachteten Thatsachen aus dem Umstande erklärt, dass der Verlust der Grosshirnlappen die Thiere verhindert, "ein objectives Bild der augenblicklichen Lage ihrer Körpertheile mit dem subjectiven der normalen Lage zu einer gemeinsamen Anregung zu verbinden". So behalten die Thiere im Zustande der Ruhe

1) Schiff, Lehrbuch der Physjologie. S. 339. 
selbst anomale Stellungen der Extremitäten bei und können, einmal angeregt, den Zustand der ihnen ertheilten Bewegung nicht mehr selbständig verlassen, bis entweder die Kraft oder alle Erregung völlig geschwunden ist. Während bei diesen Untersuchungen behufs Erreichung des Streifenhügels der Grosshirnmantel in grossem Umfange abgetragen worden war, versuchte weiterhin Nothnagel durch Einspritzung kleinster Mengen von Chromsäure localisirte Ausfallsheerde in den tiefer gelegenen Gehirntheilen zu erzeugen, ohne dass also hierbei erhebliche Läsionen der dieselben deckenden Theile des Hirnmantels gesetzt wurden. In einer Reihe anderweitiger Versuche wurde derselbe Effect durch Eingehen mit einer feinen Nadel, mit welcher kratzende Bewegungen in der Tiefe ausgeführt wurden, von Nothnagel zu erzielen versucht. Diese Untersuchungen, welche völlig methodisch an den einzelnen Abschnitten des Gehirns von Kaninchen geführt wurden, sind in Bd.57, 58,60, 62, 67 dieses Archivs mitgetheilt. Nach diesen Versuchen kommt Nothnagel zu dem Ergebniss, dass die Verletzungen des Nucleus caudatus zwei gesonderte Erscheinungen zu erzeugen vermögen, einmal einen unbezwinglichen Trieb des Thieres zum Laufen, wenn eine ganz umschriebene Stelle des Nucleus caudatus, welche dem freien dem Ventrikel zugekehrten Rande desselben nahe gelegen ist, getroffen wird, die eben deshalb von Nothnagel den Namen des Nodus cursorius erhielt, und andererseits ausgesprochene Motilitätsstörungen, welche sich insbesondere in eigenthümlichen Deviationen der Beine beim Laufen kundgaben, wenn grössere Partien des Streifenhügels verletzt wurden. Ueberleben die Thiere die Operation lange genug, so können beide Gruppen von Symptomen sich wieder zurückbilden. - Diese Angaben Nothnagel's stiessen vielfach auf Widerspruch. Carville und Duret, Schwahn und Eckhard ${ }^{1}$ ) bestätigen den Nodus cursorius überhaupt nicht, während Bechterew ${ }^{2}$ ) die Laufbewegungen bei isolirter Zerstörung der vorderen Abschnitte der centralen grauen Substanz des 3. Ventrikels, auch ohne die geringste Zerstörung des Streifenhügels beobachtete. - Bechterew hegt sonach kei-

1) Hermann, Handbuch der Physiologie. 1879. Bd. II. Th. 2. S. 132.

2) Bechterew, Zur Physiologie des Knochengleichgewichtes. Archiv f. d. gesammte Physiologie. Bd. 31. S. 509. 
nen $Z$ weifel, "dass das Corpus striatum in der Hervorbringung äieses Symptomes keine wesentliche Rolle spielt; doch scheint es ihm ganz plansibel, dass die nahe Nachbarschaft genannter Ganglien mit dem vorderen Abschnitt des Ventrikels bei Operationen am Corpus striatum zu Mitverletzung eines 'Theiles des vorderen Abschnittes der centralen Substanz (des Ventrikels) führt, als Resultat welcher in diesen Versuchen die zwangsweisen Laufbewegungen nach vorn sich ergeben konnten". - Während also auf diesem Gebiete die Angaben der Autoren vielfach widersprechend waren, kamen gelegentlich der von anderen Gesichtspunkten aus unternommenen Untersuchungen neue Thatsachen zum Vorschein, welche die Function des Corpus striatum aufzuklären im Stande zu sein schienen. - So hatten Aronsohn und Sachs ${ }^{1}$ ), $0 \mathrm{tt}^{2}$ ) und Richet ${ }^{3}$ ) den Nachweis erbracht, dass Verletzungen des Corpus striatum von Temperatursteigerungen mit dem Charakter der Fiebertemperaturen gefolgt waren. -

Alle diese, zum Theil einander widersprechenden, zum Theil neuen Resultate machten es wünschenswerth, die Untersuchungen über die Function des Nucleus caudatus neuerdings wieder aufzunehmen und womöglich mit Operationsmethoden vorzugehen, welche eine ganz umschriebene und völlig genaue Localisation der Verletzung gestatten, ohne dass, dies vorausgesetzt, der die Centralganglien deckende Hirnmantel irgend mehr verletzt wurde, als unumgänglich nöthig war. - Wir hatten überdies die Absicht, in Verfolg der von Soltmann an der Grosshirnrinde ausgeführten Untersuchungen an neugebornen Thieren, die Studie für das central gelegene Corpus striatum an eben solchen weiterzuführen, gaben indess diese Absicht auf, nachdem wir erkannten, dass schon an erwachsenen Thieren die Mannichfaltigkeit der zu Tage tretenden Erscheinungen erheblich war und za vielfachen Ueberlegungen Anlass gab. Wir concentrirten schliesslich unser Bestreben dahin, die sorgfältig operirten Thiere möglichst lange am Leben zu erhalten, um die Beobachtungsdauer möglichst ausdehnen zu können. - Zu Versuchsthieren

1) Pflŭger's Archiv Bd. 37 .

2) American Journal of nervous diseases. 1844 und Centralbl. f. med. Wissenschaft. No. 43. 1885.

3) Compt. rend. XCVIIT. p. $\$ 27$. 
dienten uns sonach zumeist ziemlich starke, erwachsene Kaninchen; nur wenige Versuche wurden an jungen Katzen gemacht. - Wenn man am Kaninchenschädel von vorn nach hinten gehend, die Sutura sagittalis verfolgt, so findet man fast senkrecht auf diese stossend die Sutura coronaria. Genau in den so gebildeten vorderen rechten Winkeln befindet sich rechts und links das Operationsfeld, von dem aus es möglich ist auf dem kürzesten Wege das Corpus striatum zu erreichen. Denkt man sich jeden der rechten Winkel halbirt und auf der die Winkel von $45^{\circ}$ abtheilenden Halbirungslinie vom Scheitelpunkt aus $2,5-3 \mathrm{~mm}$ abgetragen, so ist der so bezeichnete Punkt derjenige, von welchem aus es gelingt, mittelst einer senkrecht in die Hirnmasse eingestossenen Nadel den vordersten Theil des Nucleus caudatus, da wo derselbe wulstartig in den Ventrikel frei hineinragt, zu treffen. - Die von uns geübte vorbereitende Methode bestand in Folgendem. - Nach sorgfältiger Entfernung der den mittleren Theil des Schädels bedeckenden Haare und sorgfältiger Desinfection der Haut mittelst $(1 \%)$ Sublimatlösung wurde mit antiseptisch gehaltenem Messer und bei strenger Innehaltung anderweitiger antiseptischer Cautelen die Haut durchtrennt, alsdann gerade in dem Scheitel der beiden rechten Winkel das Periost durch einen $\perp$ Schnitt eingeschnitten und die so gebildeten Zipfel rechts und links zurückgeschlagen. In einem der beiden, oder nach Bedürfniss in beiden jetzt genau sichtbaren rechten Winkeln wurde so, dass die Nähte nahezu die Tangenten des kleinen Kreises bildeten, ein $8 \mathrm{~mm}$ im Durchmesser haltender Trepan eingesetzt und je ein kreisförmiges Trepanloch angelegt. Die Operation geschah anfänglich in Chloralnarkose, später, in der Absicht das Verhalten der Thiere sofort nach der Operation zu studiren, ohne Narkose. Während der Trepanation zeigten sich bei einzelnen Thieren Kaubewegungen, die sofort schwanden, nachdem das losgelöste kleine kreisförmige Knochenstück entfernt war. Weiterhin wurde nun die Dura gespalten und so das Gehirn völlig unversehrt blossgelegt. - Bei einer Anzahl der von uns zuerst operirten Thiere versuchten wir nun durch Einstechen Carlsbader Nadeln bis zu einer von uns an todten Kaninchengehirnen festgestellten angemessenen Tiefe eine Verletzung des Corpus striatum zu setzen, von welcher wir 
hofften, dass dieselbe, wenn wir anders mehrere Nadeln, welche neben einander eingeführt wurden, stecken liessen, eine Zerstörung des Nucleus caudatus herbeiführen würde. Die Zerstörung glückte auf solche Weise in der That, beschränkte sich indess nicht auf den Nucleus caudatus, sondern dehnte sich auf den Nucleus lentiformis aus; auch blieben die Thiere für unsere Zwecke nicht lange genug nach der Operation am Leben, wenngleich sie zuweilen 14 Tage die Einführung der ersten Nadel, auch wenn in der Zwischenzeit neuerdings Nadeln eingefïhrt wurden, überstanden. - Die Operationsmethode wurde aus den bezeichneten Gründen verlassen, und es wurde versucht durch Einsenkung von kreisförmig scharfgeschliffenen Metallhülsen von 1-3 $\mathrm{mm}$ Durchmesser, die in das Gehirn eingesenkt, und entweder sofort entfernt oder eine Zeit lang stecken gelassen wurden, eine Vernichtung des Nucleus caudatus zu erzielen. -- Auch diese Operationsmethode entsprach indess nicht unseren Wünschen und zwar um so weniger, als blutige Infiltration des Gehirns in der Umgebung der ausgestanzten Partie und rasch eintretende Entzündung Nebenverletzungen setzte, welche einen Schluss auf die Function des direct in Angriff genommenen Gehirntheils ausschlossen. Die so operirten Thiere ïberlebten überdies den Eingriff fast nie über 8-9 Tage, - Ebenso wenig entsprach eine dritte Operationsreihe, bei welcher wir uns der Bohrmaschine bedienten, unseren Anforderungen, aus den gleichen Gründen. -

Alle diese Schwierigkeiten wurden durch eine Operationsmethode überwunden, welche einer von uns (Dr. Lehmann) nach Versuchen am todten Kaninchengehirn vorsehlug, und welche sich in der Folge am lebenden Thiere vortrefflich bewährte. Armirt man den Saugeschlauch einer Wasserstrahlluftpumpe, wie sie vielfach in chemischen Laboratorien verwendet wird, mit einem in eine feine Spitze ausgezogenen Glasrohre, so ist man im Stande mittelst derselben bei hergestelltem Vacuum die Gehirnmasse an beliebiger und ganz circumscripter Stelle abzusaugen. Man hat es in der Hand die Grenzen des zu entfernenden Gehirntheiles auf das Genaueste innezuhalten, ohne dass eine Nebenverletzung erfolgt. - Die Erfahrung lehrte überdies, dass die Operationsmethode eine so sorgfältige Reinigung der 
Gehirnwunde von Blut und Detritus gestattete, wie keine andere; sie war auf solche Weise sehr dazu geeignet die antiseptischen Cautelen bei der Operation zu unterstützen. - Nach dem Absaugen des Gehirntheiles wurde die Wunde mittelst sterilisirter gekühlter physiologischer Kochsalzlösung, die tropfenweise eingelassen wurde, so lange gespült, als die Flüssigkeit überhaupt noch blutig erschien, wobei die Waschflüssigkeit fortdauernd abgesogen wurde. - Die Schliessung der Wunde geschah alsdann ebenfalls unter antiseptischen Cautelen, durch Nähte, über welche ein Jodoformverband befestigt wurde. - Die Methode gestattete es begreiflicherweise auch bei einem und demselben Thiere in mehreren Sitzungen nach und nach immer weiter fortschreitende Läsionen des Gehirnes zu setzen, ohne dass irgend welche accidentelle Entzündungen in den benachbarten Gehirntheilen die Beobachtungen störten. Sie bewährte sich endlich ganz vorzüglich für die Intention, die Thiere recht lange am Leben zu erhalten, da sie nach hinreichend gewonnener Uebung nahezu als völlig ungefährlich erschien, von den Thieren auch nach mehrfachen Eingriffen viele Monate hindurch überdauert wurde und eine in unser Belieben gegebene Beobachtungsdauer gestattete.

\section{Operationen an der Hirnrinde.}

Entfernt man bei einem Kaninchen ohne Anwendung der Narkose an der oben bezeichneten Stelle des Grosshirns auf einer Seite mittelst des Absaugeröhrchens die graue Hirnrinde bis auf das weisse Marklager, indess möglichst ohne letzteres anzugreifen, kreisförmig im Durchmesser von $8 \mathrm{~mm}$, so beobachtet man wenige Minuten nach der Operation Folgendes: Das Thier zeigt gegenüber dem früheren Verhalten eine auffällige Veränderung seines Wesens. Es erscheint überaus lebhaft, die Ohren hoch aufgesetzt blickt es scheu nach allen Seiten und schrickt bei leisen Geräuschen auf. Sich selbst überlassen putzt es wohl zuweilen, anf den Hinterbeinen sitzend, mit den Vorderpfoten völlig geschickt, aber augenscheinlich lebhafter als ein gesundes Thier Schnauze und Gesicht. - Versucht man sich dem Thiere zu nähern, so beginnt dasselbe in grossen Sätzen davon zu eilen, wobei sich der völlig geschickte und ungestörte Gebrauch aller Extremitäten herausstellt. Bei dem Versuche das Thier zu 
haschen wird man von der ausserordentlichen Lebhaftigkeit und Regsamkeit des Thieres überrascht. Nach rechts und links in geschicktester Weise ausweichend, dabei entgegenstehende Gegenstände ebenso geschickt und selbst im eiligsten Laufe vermeidend ist es fast für einen Einzelnen unerreichbar, dasselbe macht so auf Jedermann den Eindruck eines völlig normalen, sehr lebhaften Thieres. - Wird das Thier sogleich nach beendeter Operation ruhig auf den Tisch gesetzt, so hält es die normale Haltung der Extremitäten nur für kurze Zeit inne; alsbald beobachtet man indess, dass das Thier mit den Extremitäten der contralateralen Seite und zwar ebensowohl mit der vorderen, wie mit der hinteren Extremität, mit der ersteren allerdings auffälliger, auf der Unterlage ausgleitet. Die durch das Weggleiten der Extremitäten augenscheinlich unbequeme Lage hält das Thier eine Zeit lang inne und zieht die Extremitäten erst spät in die normale Position zurück, wenn nicht besondere Anregung dazu gegeben wird. - Macht man den Versuch, die der Operationswunde contralaterale Vorderpfote nach vorn zu ziehen oder nach seitwärts zu ziehen und in gestreckter Haltung liegen zu lassen, oder legt man die Pfote dorsal flectirt auf, so lässt das Thier, welches doch sonst so lebhaft erschien, alle diese Manipulationen ohne Widerstreben mit der Extremität vornehmen. Die Vorderextremität bleibt in anomaler Haltung liegen und wird erst spät in die normale Stellung wieder zurückgezogen. Dasselbe Manöver gelingt an der gleichseitigen Hinterextremität, wenngleich nicht so gut, wie vorn. - Der gleiche Versuch auf der anderen (der Operations-) Seite führt nur dazu, dass das Thier jeden Angriff auf die Extremität mit raschestem Zurückziehen derselben in die normale Lage beantwortet. Auffällig ist bei diesen Versuchen noch die Erscheinung, dass die Muskeln der soeben als geschädigt gekennzeichneten Extremitäten eine bei Weitem geringere Spannung zeigen als diejenigen der normalen Extremitäten; dieselben bieten beim Versuch die Extremitäten zu bewegen, einen auffallend geringeren Widerstand dar, als die Muskeln der anderen Seite. Hat man die geschädigten Extremitäten in die geschilderte anomale Lage gebracht und überlässt das Thier jetzt sich selbst, so vergeht sehr geraume Zeit bevor das Thier spontan dieselben wieder normal stellt: man kann indess beobachten, dass das 
Thier die Extremitäten sofort und plötzlich wieder in normale Haltung bringt, wenn man die ausgestreckte Extremität kneift oder drückt. - Vorausgesetzt, dass völlig aseptisches Verhalten der $W$ unde vorhanden ist, bleiben die Thiere fast immer völlig fieberfrei; nur ganz vereinzelt wurden für wenige Stunden geringe Temperatursteigerungen beobachtet, besonders bei solchen Thieren, welche zum $Z$ wecke der Operation narkotisirt worden waren und deren Temperatur während der Operation und einige Zeit darauf subnormal geworden war. - Das Verhalten der Respiration ist bei den nicht narkotisirten Thieren sofort nach beendeter Operation normal, auch fressen die Thiere alsbald spontan. - Wenige Tage schon nach der Operation lässt die geschilderte Erregtheit der Thiere nach, und auch die an den Extremitäten beobachteten Phänomene beginnen allmählich zu verschwinden. Bei einzelnen Thieren konnte schon am 7. Tage nach der Operation nur wenig mehr davon wahrgenommen werden und bei solchen Thieren, welche wir längere Zeit hindurch beobachteten, war schliesslich jede Spur der geschilderten Erscheinungen verschwunden. Die Thiere konnten von völlig intacten in keiner Weise unterschieden werden. Wurde die Operation an genau der gleichen Stelle des Gehirns beiderseits gemacht, so zeigten sich, neben den Erregtheiten der Thiere, die beschriebenen Phänomene beiderseits, wenngleich es allerdings nicht gelang, dieselben beiderseits völlig gleichmässig zum Vorschein zu bringen; immer erschienen die Störungen an der einen Seite, wechselnd ohne Regelmässigkeit, rechts oder links, stärker ausgeprägt, als an der anderen. Aber auch bei den beiderseits operirten Thieren erfolgte nach einiger Zeit eine vollständige Rückkehr zum normalen Verhalten; auch diese Thiere konnten bei längerer Beobachtungsdauer von normalen nicht unterschieden werden.

Bei der Section dieser Thiere konnte mit Ausuahme von einer flachen, mit Dura und Haut zusammenhängenden narbigen Einziehung an der operirten Stelle der Hirnrinde etwas Abnormes im Gehirn nicht nachgewiesen werden.

Unsere Beobachtungen bestätigen, soweit es sich um einseitige Operationen an der Hirnrinde handelt, im Wesentlichen die von Nothnage ${ }^{1}$ ) angegebenen Thatsachen; wir können den-

1) Dieses Archiv Bd. 57. S. $192 \mathrm{ff}$. 
selben nur noch die eigenthümliche Verringerung der Muskelspannung an den geschädigten Extremitäten hinzufügen, welche sich also wesentlich darin äussert, dass die Extremitäten mit grosser Leichtigkeit und fühlbar herabgesetztem Widerstand in den Muskeln sich in anomale Lagen bringen lassen. - Wird das Ueberlassen der Extremitäten in anomaler Haltung, wie von Nothnagel geschieht, als partielle Lähmung des Muskelsinnes gedeutet, so handelt es sich also nebenbei augenscheinlich um Störungen der Muskelinnervation im motorischen Sinne oder man müsste annehmen, dass in Folge der verminderten Sensibilität eine unter normalen Verhältnissen auf jedes Anfassen eintretende reflectirende Spannung der Musculatur ausbleibt. Weiterhin hebt Nothnagel wohl hervor, dass die Thiere normalen gleichen, munter aussehen und wie gesund umherspringen, er charakterisirt indess nicht den ganz augenscheinlich hervortretenden hohen Erregungszustand der Thiere, welcher sich in lebhafter Reaction auf sensible Reize ebensowohl wie auf Sinnesreize, insbesondere auf Gesichts- und Gehörswahrnehmungen kund giebt, vielmehr nimmt Nothnagel diese letztere Erscheinung für die Verletzungen des Nucleus caudatus in Anspruch, was also nach unseren Beobachtungen nicht zutrifft, da bei unseren Thieren eine Verletzung der tiefer gelegenen Hirntheile völlig ausgeschlossen war. Eine höehst beachtenswerthe Aehnlichkeit zeigten unsere Thiere in ihrem Wesen mit denjenigen, welche Goltz am vorderen Quadranten des Grosshirns operirt hatte. Wenn es uns erlaubt ist, unsere Kaninchen mit den von Goltz operirten Hunden zu vergleichen, so zeigten beide Gruppen von Thieren eine auffallend gesteigerte Erregbarkeit gegen Gesichts- und Gehörseindrücke und eine gesteigerte Lebhaftigkeit des gesammten Verhaltens. Ferner ist uns also geglückt, was Nothnagel nicht gelungen war, beiderseits operirte Thiere Monate lang am Leben zu erhalten und auch hierbei völligen Ausgleich der gesetzten Störungen zu beobachten, wir könnten somit die von Nothnagel aufgeworfene Frage, ob nach Entfernung beiderseits gleich gelegener Partien der Hirnrinde andere dafür vicariirend in Function treten, in bejahendem Sinne beantworten; indess scheint uns auch noch eine andere Erklärung nicht ausgeschlossen. 
Operationen an der grauen Hirnrinde und der darunter liegenden weissen Substanz des Gehirns ohne Eröfnung des Ventrikels.

Wenn bei der vorbereitenden Operation, der Trepanation des Schädels an der bezeichneten Stelle zufälliger Weise und unbeabsichtigt ein etwas stärkerer Druck mit dem Trepan ausgeübt wurde, so konnte die Wahrnehmung gemacht werden, dass zunächst mimische Zuckungen bei den Kaninchen auftraten, welche alsbald von ausgesprochenen Kaubewegungen gefolgt waren. Die Kaubewegungen sistirten sofort, wenn das von den übrigen Schädelknochen losgelöste kreisförmige Knochenstück entfernt wurde, sie traten auch nicht wieder auf, so lange man bei dem Absaugen des Gehirns noch graue Hirnrinde entfernte; dieselben erschienen indess fast ausnahmslos wieder, wenn nach Absaugung der grauen Rindensubstanz die darunter liegende weisse Substanz des Gehirns mit dem Absaugeröhrchen in Angriff genommen wurde; gleichzeitig beobachtete man eine seitliche Wendung des Kopfes nach der Operationsseite hin. Die Erscheinungen kamen an demselben Thiere vor, sowohl, wenn rechts oder links operirt wurde, gleichviel ob bei beiderseitiger Operation in einer Sitzung beide Seiten operirt wurden oder ein mehrtägiger Zeitraum dazwischen lag. - Kaubewegungen, Nackencontractur und Wendung des Kopfes nach der Operationsseite verschwinden indess sobald die oberste Schicht des weissen Marklagers entfernt ist, und treten auch nicht wieder auf, wenn man sich der grauen Decke des Ventrikels mit dem Absaugeröhrchen nähert. - Wurde nun die Operation beiderseits so ausgeführt, dass der Ventrikel nicht eröffnet wurde, unverletzt blieb, - und man hatte an dem Auftreten der respiratorischen Schwankungen der Ventrikelflüssigkeit, welche sich an der Decke des Ventrikels markirte, eine gute Orientirung für das Vorgehen bei der Entfernung der weissen Substanz - so zeigten die so operirten Thiere folgende Erscheinungen. Die Thiere verhielten sich genau ebenso, wie diejenigen, welche nur an der Hirnrinde operirt worden waren. Dieselbe Erregbarkeit in Wesen, dieselben Phänomene an den Extremitäten. Es gelang bei beiderseitig operirten Thieren alle 4 Extremitäten in völlig sonderbare 
und anomale Lagen zu bringen, ohne dass die Extremitäten spontan in normale Haltung zurückgebracht wurden; erst in dem Augenblicke, wo eine der so fehlerhaft gelagerten Extremitäten gedrïckt warde, wurde zunächst diese, plötzlich und rasch wurden aber auch die anderen in normale Lage zurïkgebracht. Auffällig ist auch hier die wesentlich verringerte Muskelspannung in den Extremitäten. Das Verhalten der Thiere glich also vollkommen demjenigen, welches Schiff von solchen schildert, denen er nach Entfernung der Hirnlappen die beiden Streifenhügel entfernt hatte ${ }^{1}$ ). Auch im Weiteren ist das Verhalten unserer Thiere demjenigen gleich, welche die von Schiff operirten Thiere zeigten. - Dieselben zeigen ebenso wenig, wie jene irgend ausgesprochene Lähmungen bei der Locomotion. Das Laulfen erfolgt, wenn die Thiere durch Kneifen des Schwanzes oder in anderer Weise dazu angeregt werden, anscheinend völlig normal, mit völlig zweckmässigem Gebrauch aller 4 Extremitäten genau in der Weise, wie es von gesunden Kaninchen geschieht. Auch diese tiefer gehenden Operationen verlaufen bei völlig aseptischer Behandlung der Wunden ohne wesentliche Temperatursteigerung und nur während der Operation narkotisirte Thiere, deren Temperatur sich zuweilen bis $34,5 \mathrm{C}$. herabminderte, zeigten einige Zeit nach der Operation ein Ansteigen der Temperatur bis auf 40,5 oder 41,2 welche indess in wenigen Stunden der Normaltemperatur von 39,6 wieder Platz macht. - Die Widerstandsfähigkeit der Thiere gegen die Operation ist genau die gleiche, wie gegen die früher beschriebene mehr oberflächliche, so dass an diesen Thieren einige Zeit nach dem ersten Eingriff die tiefer dringende Operation vorgenommen werden konnte. Gerade aus diesem Grunde fehlen uns über die Restitution der gesetzten Störungen die Beobachtungen, nur soviel sind wir im Stande auszusagen, dass dieselben 5 Tage nach der beiderseitigen Operation noch bestanden. Wir werden übrigens bezüglich der Restitution aus den weiteren Mittheilungen auch für die in Rede stehende Kategorie von Operationen Schlüsse ziehen können.

1) Sehiff, Lehrb. d. Physiologie. Bd. I. S. 339. 


\section{Operationen mit Eröffnung der Ventrikel.}

Die Eröffnung der Ventrikel wurde bei zwei Gruppen von Thieren vorgenommen, einmal bei solchen, welche schon vorher bis auf die Ventrikeldecke operirt waren und eine Zeit lang beobachtet wurden, sodann aber auch bei völlig intacten Thieren, denen an der bezeichneten Stelle die Gehirnmasse bis zur Eröffnung des Ventrikels durch Absaugen entfernt wurden. Für beide Gruppen von Thieren gilt das Gleiche, dass auch die beiderseitig vorgenommene Eröffnung der Ventrikel und der $\mathrm{Ab}$ fluss von Cerebrospinalflüssigkeit an sich so gut wie gar keinen Effect hervorruft. Weder treten Zuckungen auf, noch anderweitige besondere Bewegungserscheinungen; die Thiere verhielten sich vielmehr auch ohne Narkose während der Operation ruhig, wie die früher operirten. Augenscheinlich ist die Eröffnung des Ventrikels schmerzlos, dieselbe ist in der Regel weder sofort nach der Operation, noch auch in der Folge von Steigerung der Körpertemperatur gefolgt, wenngleich eine solche in vereinzelten Fällen vorkommt, und zwar mit Wahrscheinlichkeit dann, wenn auf die durch die Eröffnung des Ventrikels freigelegten centralen Gebilde zufällig ein besonderer Reiz ansgeübt worden ist, wovon weiterhin noch die Rede sein wird. - Die Thiere, welchen die Hirnventrikel eröffnet worden sind, zeigten im höchsten Grade das oben skizzirte lebhafte Wesen. Die Ohren hoch aufgesetzt, den Kopf leicht rückwärts gebogen, achten sie auf das geringste Geräusch, blicken scheu um sich und vermeiden jede Annäherung, indem sie in grossen Sätzen davon springen, entgegenstehenden Gegenständen in geschicktester Weise ausweichend. Die Haltung der Extremitäten erscheint im Laufen intact; das Sehvermögen ist augenscheinlich normal. - Zum Laufen angeregt, halten die Thiere nach einigen lebhaften Sprüngen spontan wieder inne, so dass von einem inneren Triebe der Thiere zum Laufen keine Rede sein kann. - Das Verhalten der Extremitäten ist, wenn anders die Thiere nur einseitig operirt worden sind, an der contralateralen Seite genau so, wie es oben für die Rindenoperationen beschrieben worden ist, ebenso bei den beiderseitig operirten Thieren, deren sämmtliche 4 Extremitäten in beliebig abnorme Lage gebracht werden können, ohne dass 
dieselben in die normale Position zurückgeführt wurden. Dies geschieht, wenn man eine der Extremitäten drückt oder kneift, dann aber plötzlich und rasch und an allen 4 Extremitäten. Auch die Verminderung der Muskelspannung giebt sich sehr deutlich kund. - Eine Rückbildung der Erscheinungen wurde noch nach 10 Tagen nicht beobachtet, selbst die intensive Erregbarkeit im Wesen der Thiere konnte nach dieser Zeit noch beobachtet werden und bei einem einzelnen Thiere, welches durch Diarrhöen so stark heruntergekommen war, dass es am 13. Tage getödtet wurde, war bis zum letzten Augenblicke die mit seinem elenden Körperzustand auffällig contrastirende Lebhaftigkeit des Wesens bemerkenswerth. - Dass indess auch bei den mit Eröffnung des Ventrikels operirten Thieren schliesslich eine völlige Rückbildung aller Erscheinungen folgt, ergiebt sich aus dem Folgenden.

Eröffnung der Ventrikel mit nachfolgender Reizung des Corpus striatum (Nucleus caudatus).

Nach der Eröffnung der Ventrikel und Absaugung der Ventrikeldecke im Umfange der Trepanationsöffnung präsentiren sich als frei in die Ventrikel hineinragend jederseits zwei wulstförmige Gebilde, mehr nach vorn und lateralwärts der Kopf des Corpus striatum (Nucleus caudatus) nach rückwärts und medianwärts der Thalamus opticus. - Das Corpus striatum liegt je in seinem vordersten Abschnitte völlig zu Tage und die an ihm vorzunehmenden Eingriffe können ohne Gefahr der Nebenverletzungen vorgenommen werden. Bevor wir nun zur definitiven Entfernung desselben schritten, versuchten wir an einigen Thieren festzustellen, welche Erscheinungen von der einfachen mechanischen Reizung desselben ausgelöst werden konnten. Wir hofften auf diesem Wege am ehesten den, wie oben erwähnt, von Nothnagel festgestellten, von Carville und Duret'), Eckhard und Schwahn und auch von Bechterew bestrittenen Nodus cursorius feststellen zu können. Es wurde also nach Freilegung des Nucleus caudatus möglichst genau an die von Nothnagel bezeichnete Stelle eine Carlsbader Nadel eingestochen und nach einigen Secunden entfernt. Es kann zunächst hervorgehoben werden, dass das von

1) Carville et Duret, Arch. de physiologie et pathologie. 1875 . 
Nothnagel beschriebene Phänomen des unaufhaltsamen zwangweisen Laufens bei den Thieren niemals zum Vorschein kam, vielmehr verhielten sich dieselben genau ebenso, wie die früher geschilderten, so dass hier nichts Neues hinzugefügt werden kann. Die Sensibilität der Haut war wie bisher intact, die Störungen an den Extremitäten sind genau die früher beschriebenen. Aber eine neue Erscheinung trat regelmässig auf, vou welcher bei den früheren Operationen nur hin und wieder Andeutungen vorhanden gewesen waren, d. i. eine der Operation regelmässig folgende rapide Temperatursteigerung in der Weise, wie Aronsohn und Sachs dieselbe beschrieben haben. Die Temperatur stieg innerhalb weniger Stunden nach der Operation rasch an und erhob sich bis $41,6^{\circ} \mathrm{C}$, im Anus gemessen, blieb mit geringen Schwankungen bis etwa 4 Tage auf dieser Höhe und sank dann allmählich zur Norm zurück. - Es ist oben erwähnt worden, dass in einzelnen Fällen die einfache Eröffnung des Ventrikels von einer ähnlichen Temperatursteigerung gefolgt war, so beobachteten wir nach dieser Operation einmal schon 8 Stunden nach dem Eingriff ein Ansteigen der Analtemperatur von $39,7-40,3^{\circ} \mathrm{C}$. in weiteren Stunden bis $41,3^{\circ} \mathrm{C}$.; indess sank dieselbe innerhalb der nächsten 24 Stunden auf 40,3 ab und ging dann zur Norm zurück. In Zusammenhange mit den eben wiedergegebenen Beobachtungen kann jetzt die Vermuthung ausgesprochen werden, dass in diesem Falle und ähnlichen Fällen eine uns unbekannt gebliebene Reizung der vorderen Abschnitte des Nucleus caudatus gelegentlich der Eröffnung des Ventrikels eingetreten war.

Die mit Eröffnung beider Ventrikel und überdies noch durch Nadelstiche in die Corpora striata operirten Thiere vermochten wir recht lange am Leben zu erlalten, und es zeigte sich, dass innerhalb 2 Monate eine nahezu vollständige Restitution der gesetzten Störungen eintrat. Zunächst verlor sich die übergrosse Erregbarkeit und Schreckhaftigkeit der Thiere, während das anomale Verhalten der Extremitäten vorläufig bestehen blieb. Allmählich verlor sich indess auch dies; die Extremitäten verblieben nicht mehr in anomal gelegten Stellungen, sondern wurden schliesslich genau so exact, wie von einem gesunden Thiere zurückgeführt. Weder beim I,aufen, noch auch sonst in dem 
Verhalten der Thiere war man bei sorgfältiger Beobachtung im Stande irgend welche Anomalien zu entdecken, welche dieselben von gesunden hätten unterscheiden lassen. Die Section des Gehirns ergab, dass der Knochendefect am Schädel von einer narbigen Bindegewebsmasse eingenommen wurde, welche mit dem Gehirn in Zusammenhang war. Am Gehirn selbst zeigte sich eine geringe narbige Einziehung an der Operationsstelle. In den durch Narbenmasse von oben verschlossenen Ventrikeln war ziemlich reichlich Flüssigkeit vorhanden und am Kopfe des Nucleus caudatus sah man ganz geringfügige flache Vertiefung; im Uebrigen erschien das Corpus striatum, ebenso wie das ganze übrige Gehirn intact.

Zerstörung der Nuclei caudati im grössten Umfange nach Eröffung der Ventrikel.

Die Anwendung ziemlich fein ausgezogener Saugeröhrchen gestattete nach Eröffnung der Ventrikel nicht allein die Entfernung des frei liegenden Kopfes der Nuclei caudati, sondern auch die des grössten Theiles der lang sich rückwärts erstreckenden Cauda derselben. Gleichzeitig wurden durch das Absaingen von Blut und etwaigen geringen Detritusmassen die durch Ansammlung und Liegenbleiben derselben etwa entstehenden Nebenerscheinungen ausgeschlossen. Dies war begreiflicherweise bei der von Nothạagel geübten Methode der Zerstörung des Gebildes durch Nadeln nicht zu bewerkstelligen, auch gestattete unsere Operationsmethode ein Ueberblicken wenigstens eines beträchtlichen Theiles des Operationsfeldes. -

Wurde die Operation auf einer Seite ausgefuhrt, so zeigten die ohne Narkose operirten Thiere kurze Zeit nach derselben folgende Erscheinungen. - In erster Linie beobachtet man die uns nunmehr schon hinreichend bekannte und zwar jetzt excessiv gesteigerte Erregbarkeit, die sich ebenso sehr gegen Sinnesreize kundgiebt, wie in dem Widerstreben der Thiere hervortritt, sich haschen zu lassen; jedoch besteht jetzt so wenig wie früher ein ausgesprochener innerer Lauftrieb. Sehvermögen und Gehörsempfindungen erschienen intact. - Die Störungen in den contralateralen Extremitäten sind stark ausgeprägt. Dieselben gleiten auf der Unterlage aus und bleiben in den per- 
versen Positionen in welcher sie schliesslich gerathen oder absichtlich gebracht werden. - Die Störung in der Spannung der Muskeln ist sehr deutlich. Die Thiere laufen und hüpfen zwar, wenn zum Laufen angeregt, wie alle bisher beschriebenen, man beobachtet indess und zwar vorzugsweise an der contralateralen vorderen Extremität eine ausgesprochene Störung der Beweglichkeit. Die contralaterale Vorderpfote stellt sich, während der gesammte Vorderkörper ein wenig nach der contralateralen Seite hinneigt, nach innen unter den Leib, und bleibt während des Jaufens in der Action ein wenig zurück, wenigstens glaubte der Eine von uns diese Thątsache sicher constatiren zu können. Die Erscheinung ist beim Sitzen des Thieres ebenfalls deutlich, wobei die Vorderpfote besonders nach innen unter Brust und Bauch gestellt verharrt. Die Steigerung der Körpertemperatur ist zwar deutlich, aber nicht sehr erheblich, währt auch in der Regel nicht lange, sondern sinkt schon am Tage nach der Operation zur Norm zurück; sie ist jedenfalls geringer als diejenige, welche durch einfache Reizung des Corpus striatum erzeugt wird und von welcher die Rede war. - In einigen Fällen schien es, wie wenn an der contralateralen Seite auch die Sensibilität ein wenig herabgesetzt wäre, wenigstens schienen die Thiere auf leise Reizungen an den Haaren, schwache Nadelstiche etc. nieht so lebhaft zu reagiren, wie auf der anderen Seite. -

Alles in Allem beobachtet man aber nahezu dieselben. Erscheinungen, welche schon aus den früheren Operationen bekannt geworden waren; sie decken sich auch im Wesentlichen mit den von Nothnagel ${ }^{1}$ ) beschriebenen Phänomenen. - Es glückte die so operirten Thiere zum Theil recht lange am Leben zu erhalten; sie frassen spontan und zeigten bei aseptischer Behandlung der Wunde keine Störungen von dieser Seite her. - Im Verlaufe der sich bei einigen Thieren auf Monate erstreckenden Beobachtungsdauer konnte nun Folgendes constatirt werden. Die Steigerung der Körpertemperatur, wo solche überhaupt zu Stande gekommen war, wich zuerst, darauf die Erregbarkeit der Thiere. Schon nach 14 Tagen konnte an einem der Thiere, bei welchem später die Section einen fast vollständigen Verlust des

) a. a. 0. Bd. 60. S. 138.

Arehiv f. pathol. Anit. Bd. CVI. Hft.?. 
Nucleus caudatus der einen Seite nachwies, von der gesteigerten Erregbarkeit nichts mehr nachgewiesen werden. Später und langsamer glichen sich die Störungen an den Extremitäten aus, doch auch diese so vollständig, dass die Thiere in keiner Weise von gesunden mehr unterschieden werden konnten. - Wurde die Versuchsart dahin abgeändert, dass bei solchen einseitig operirten Thieren längere Zeit nach der ersten Operation und nachdem - ein gewisses Verschwinden der Erscheinungen schon stattgefunden hatte, auf der andern Seite des Gehirns die gleiche Operation der möglichst ausgiebigen Entfernung des Nucleus caudatus ausgeführt wurde, so wiederḩolten sich neben der neu auftretenden Lebhaftigkeit und Erregbarkeit des Thieres die bekannten Phänomene an der der jüngsten Operationsstelle contralateralen Seite wieder, indess konnten wir auch an der der ersten Operation contralateralen Körperseite eine Verschlimmerung beobachten. Wurden z. B. die rechten vorderen Extremitäten nach linksseitiger erster Operation wieder nahezu exact aus fehlerhaften Positionen von dem Thiere zurïckgezogen, und operirt man jetzt rechts, so stellt sich heraus, dass beide vorderen Extremitäten auf der Unterlage ausglitten und in der anomalen Position von dem Thiere belassen wurden, dass sie weiterhin beliebig selbst in Dorsalstellung gebracht werden konnten und von dem Thiere unbeachtet blieben, bis ein neuer Reiz, Kneifen oder Drücken, lie Thiere dazu bewog, die Extremitäten nunmehr rasch und ruckweise in normale Position zu bringen. - Dasselbe war, wenngleich nicht immer in gleichem, ausgiebigem Grade an den hinteren Extremitäten der Fall.

Einige Thiere wurden von uns beiderseits sofort so operirt, dass möglichst ausgiebig die Nuclei caudati entfernt wurden. Die Thiere boten ausser den uns nun schon völlig bekannten Phänomenen nichts Neues dar. Die Lebhaftigkeit der Thiere war so excessiv, dass ein Einzelner sich vergeblich bemühte, ein so operirtes Thier zu erhaschen; in raschestem Laufe, nach rechts und links geschickt ausweichend, stürmten sie, wenn sie gejagt wurden, dahin; indess auch diese Thiere beruhigten sich spontan wieder, auch sie hatten keinen inneren Trieb zum Laufen. Bei alledem waren die Störungen an den Extremitäten sehr ausgesprochen; alle glitton im ruhigen Sitzen auf der Lnterlage 
aus, alle verharrten in ihren anomalen Positionen bis ein neuer Reiz den Antrieb gab sie zurückzuziehen, bei allen war endlich eine wesentliche Verringerung der Muskelspannung vorhanden. Die Temperatursteigerung war bei einzelnen Thieren bedeutend, bei einem z. B. schon wenige Stunden nach der Operation $42^{\circ} \mathrm{C}$. und blieb auch für einige Tage hoch, um allmählich zur Norm abzusinken. -

Die beiderseits gleichzeitig operirten Thiere starben anfänglich schon nach 10 Tagen, indess gelang es uns später die Thiere länger und 1 der operirten Thiere über 8 Monate lang am Leben zu erhalten, und nach der nunmehr erreichten Uebung in Wartung und Pflege dieser Thiere scheint es uns möglich, auch fernerhin tief und beiderseits operirte Thiere in grösserer Zahl beliebig lange erhalten zu können. - Bei der so auf Wonate hin sich erstreckenden Beobachtungsdauer stellte sich heraus, dass die Thiere je länger sie am Leben blieben, desto weniger von den geschilderten anomalen Phänomenen, die nach der Operation aufgetreten waren, zeigten, bis schliesslich jede Spur derselben verschwand und die Thiere völlig normalen glichen. - Das Abklingen der Phänomene geschah auch hier in der uns schon bekannten Reihenfolge, die wir nicht mehr zu wiederholen brauchen. Das Gehirn eines dieser Thiere in sorgfältig parallele Frontalschnitte von vorn nach hinten zorlegt zeigte neben beiderseits ziemlich erheblich erweiterten Ventricularraum, welcher bei der Section mit Flüssigkeit erfüllt war, den Verlust des grössten Theiles beider Nuclei caudati, von welchen nur die hintersten Partien stehen geblieben waren. Es ergiebt sich sonach die höchst bemerkenswerthe Thatsache, dass die volle Rückbildung erfolgte, nach. dem sowohl die über den Corpora striata gelegenen Hirnpartien ebenso wie der grösste Theil der Corpora striata selbst entfernt worden waren.

Blicken wir auf die gesammte Untersuchungsreihe zurïck, so haben wir in dem.Maasse, als wir an der oben gekennzeichneten Stelle des Kaninchenhirns von aussen nach innen mit Entfernung der Gehirnmasse in der angegebenen Ausdehnung vordrangen, in steigendem Grade folgende ganz gleichmässigen Erscheinungen entstehen sehen. Eine gesteigerte Erregbarkeit der Thiere, die sich in Schreckhaltigkeit, Aengstlichkeit gegenüber 
Geräuschen, Empfindlichkeit bei Reizungen der Haut u. s. w. kund giebt. Die Neigung eiligen Laufes davon. zu stürzen, wenn man die Thiere zu haschen versucht, ohne dass indess dabei ein innerer Trieb zum Laufen sich kund giebt, da dieselben in der Ruhe verharren, wenn sie sich unbeobachtet glauben und nicht angeregt werden. Mit der Tiefe des Operationsgebietes nahm die Erregbarkeit zu und wurde am intensiysten, wenn eine Läsion beider Nuclei caudati stattgefunden hatte. Weiterhin zeigte sich eine die Extremitäten und zwar vorzugsweise die vorderen, aber auch die hinteren, betreffende Störung, die darin bestand, dass die Thiere dieselben in anomaler Lagerung liessen, in welche dieselben entweder durch Ausgleiten auf dem Bodon spontan geriethen oder durch künstliche Manipulation gebracht wurden. Auch dieses Phänomen steigerte sich mit der Tiefe des Operationsfeldes und nach Entfernung des grössten Theiles beider Corpora striata verhielten sich die Thiere so, wie Schiff und Nothnagel das beschrieben, die Extremitäten verblieben so lange in jeder beliebigen Lageveränderung bis ein ziemlich starker Reiz, wie Kneifen, Drücken etc. die Thiere zur Zurückführung der Extremitäten in die normale Haltung anregte. Eine totale Lähmung der Extremitäten bestand nicht, die Locomotion war unbehindert, wenngleich ein gewisser Grad motorischer Störung nicht wegzuleugnen ist, der sich in einer verringerten Spannung der Musculatur der betroffenen Extremitïten und bei Läsion der Corpora striata auch in einer fehlerhaften Haltung insbesondere der contralateralen vorderen Extremität beim Laufen und Sitzen documentirte. - Es muss also zugegeben werden, dass von den Corpora striata ebenso, wie von der Hirnrinde aus die motorischen Apparate beeinflusst werden, und dass die Störungen in steigendem. Maasse Statt haben, jo weiter von der Hirnrinde aus nach der Tiefe zu bis zu den Corpora striata inclusive die Läsion vordringt.

Als eine besondere Erscheinung trat bei Eröffnung der Ventrikel hin und wieder, bei Reizung der vorderen Theile der Corpora striata jedes Mal, eine Steigerung der im Anus gemessenen Körpertemperatur hervor. - Dies die Gesammtheit der Erscheinungen bei gut und exact operirten Thieren. - Dem gegenüber gelang es festzustellen, dass da, wo Nebenverletzungen der Cap- 
sula interna, des Nucleus lentiformis oder der Thalami optici stattgefunden hatten, wie dies in der Regel bei solchen Thieren der Fall war, welche mit Stanzen oder der Bohrmaschine operirt worden waren, auch anderweitige zum Theil höchst complicirte Phänomene zur Beobachtung kommen. So wurden ausgesprochene Manègebewegungen, allgemeine Convulsionen, stupides Hinbrüten mit völligem oder zeitweiligem Verlust des Vermögens spontan zu fressen, totale Lähmungen einzelner Extremitäten, Contracturen der Nackenmusculatur, intensive Verlangsamung und Vertiefung der Respiration, Krümmung der Wirbelsäule, bei solchen fehlerhaft operirten Thieren beobachtet. Wir waren an der Hand der Sectionsbefunde, welche Blutergüsse, starke Verdrängungen der den Corpora striata anliegenden Hirntheile, Anhäufung von Detritusmassen nachweisen liessen; im Stande, diese Fälle von denen nach unserer Operationsmethode rein und exact operirten Thieren zu trennen. -

Als die wichtigste Thatsache tritt uns nun an unseren gut operirten Thieren entgegen, dass man dieselben bei geeigneter Pflege lange genug am Leben erhalten kann, um eine je nach dor Lebensdauer allmähliche und, schliesslich vollständige Restitutio ad integrum zu beobachten. Die Wiederherstellung ist in dem Maasse schwieriger und nimmt längere Zeit in Anspruch, je tiefer das Operationsfeld ist, je mehr neben der Hirnrinde und dem darunter liegenden Marklager des Gehirns die eigentliche Substanz des Nuclei caudati lädirt worden ist. Indess ergiebt die Läsion der Corpora striata als Folgen im Wesentlichen qualitativ dieselben Phänomene, wie solche nach Läsion der Hirnrinde an der bezeichneten Stelle auftreten, nur quantitativ ist den Erscheinungen nach der Effect der die Corpora striata angreifenden Operation grösser, und diesem Verhältniss entspricht vollständig die für die Rückbildung in Anspruch genommene grössere Zeitdauer.

Die beobachteten Erscheinungen lassen sich unschwer in zwei Gruppen trennen, einnal tritt uns die, wie man sagen kann, rein psychische Veränderung der Thiere entgegen, die in toto gesteigerte Erregbarkeit, ihre Schreckhaftigkeit, Reizbarkeit gegenüber Sinneseindrücken u.s. w.; zweitens die möglicherweise als Ausfallserscheinungen zu deatenden motorischen Störungen, 
die Störungen des Muskelgefühls, der Abfall in der Spannung der Musculatur und geringfügige paretische Zustände in einzelnen Muskelgruppen. Da alle diese Phänomene mit der Grösse der Verletzung nach den Corpora striata zu zunehmen, und dennoch selbst nach Läsion der letzteren wieder ausgeglichen werden können, so sind wir nicht in der Lage den Corpora striata andere Functionen zuzuschreiben, als den darüber liegenden Theilen der Hemisphäre und wir können bei dieser Annahme wohl begreifen, dass die Ausfallserscheinungen nach ihrem Verlust wieder allmählich ausgeglichen werden, wie dies nach Ausfall von Partien der Gehirnrinde schon bekannt ist.

Wir gelangen auf solche Weise etwa zu folgenden Vorstellungen. Wir können annehmen, dass die Function der einzelnen sogenannten motorischen Ganglienzelle nicht eine derart fest gelegte und beschränkte ist, dass durch die von ihr ausgehenden Bewegungsimpulse stets die gleiche Reaction an dem peripherisch gelegenen Organe ausgelöst werden muss. Nicht jede Zelle und selbst nicht eine umschränktere Gruppe von Zellen wird durch Aussenden von Reizwellen in der Lage sein, stets den gleichen Muskel oder denselben Theil eines Muskels zur Contraction zu bringen. Der Effect ihrer Thätigkeit wird vielmehr ein verschiedener sein, je nach der Art und Weise, wie sie mit anderen Zellen im Augenblicke gleichsam in Actionsverbindung tritt. Wenn wir dies durch eine Art von Schema erläutern wollen, so denken wir uns den Hergang etwa folgendermaassen. Angenommen die Ganglienzellen A, B, C, D, E, F stehen unter einander in leitender Verbindung und stehen nach der Peripherie hin durch Leitungsbahnen mit verschiedenen Muskeln in Beziehung. Man kann sich dann vorstellen, dass für schwache Contractionen des Muskels a in der Regel die Ganglienzellen A, B, C in Action treten und Contractionsimpulse aussenden, für stärkere aber ausser den genannten noch D, E, F, welche ihre Impulse alsdann zum Theil nach A, B, C, zum Theil vielleicht durch vorhandene directe Bahnen nach dem zu contrahirenden Muskel direct entsenden. Die Combination der gemeinschaftlichen Thätigkeit der genannten Ganglienzellen kann in dem Einzelfalle mannichfach variiren, die von den einzelnen Zellen entwickelten Energien können bei der reichlichen Anzahl leitender Verbin- 
dungen auf verschiedenen Bahnen zur Peripherie fliessen. Alles dies vorausgesetzt, dass die Bahnen ungestört und normal erhalten sind. - Wie müssen sich nun die Verhältnisse gestalten, wenn ans der Gruppe von Ganglienzellen eine einzelne oder mehrere ausgeschaltet resp. operativ entfernt worden sind? - Die Muskeln, deren Innervation von den entfernten Zellen in der Regel, gewohnheitsgemäss und nach Uebung innervirt zu werden pflegen, werden den Ausfall der Innervation unzweifelhaft erkennen lassen. Bei Verlust von A, B, C wird also Muskel a sicher einen Ausfall in der Contraction zeigen, indess wird damit keinesweges eine vollständige Lähmung gegeben sein, sondern die Contraction wird nur, vorausgesetzt dass der gleiche psychische Reiz vorhanden ist, schwächer ausfallen, da D, E, F in die Function eintreten, und was wir an dem Muskel von Contraction wahrnehmen, ist nur der Effect der jetzt geringeren, wenngleich vorhandenen, ihm zufliessenden Impulse. - Wir haben es bei der jetzt schwächer auftretenden Coutraction des Muskels unzweifelhaft mit einer Ausfallserscheinung zu thun.Aber damit ist noch nicht gesagt, dass alle unmittelbar beobachteten motorischen Defecte unzweifelhaft derartige Ausfallserscheinungen sind. Es kommen noch weitere Momente in Betracht. - Für die erhalten gebliebenen Ganglienzellen kann es nicht irrelevant sein, dass durch gewaltsamen Eingriff ein Theil ihrer nervösen Verbindungen mit anderen gleichartigen Zellen zerrissen ist. Von der Stelle der zerrissenen Bahnen oder Verbindungen wird auch auf diese erhaltenen Ganglien unzweifelhaft ein Reiz ausgeübt, der an sich schon die Thätigkeit auch dieser Ganglienzellen modificiren muss und dieser Reiz kann ebenso erregend, wie lähmend auf ihre Thätigkeit wirken. Beides ist je nach der unmittelbaren oder mittelbaren Verbindung, näheren oder entfernteren Lage des Reizortes gegenüber der in Betracht gezogenen Ganglienzelle möglich. Es ist begreiflich, dass demnach die erwähnte, an dem Muskel beobachtete geringere oder geschädigte Contractionsenergie zum Theil auch der so eingetretenen Actionshemmung der noch erhaltenen Ganglien ihren Ursprung verdankt, und dass dieser Theil wieder sich verliert in dem Maasse, als durch Abklingen des Reizes bei Heilung der gesetzten Wunde die Ganglienzelle gleichsam ihre Actionsfreiheit 
und -Fähigkeit wieder erhält. Die in dem zu innervirenden Muskel zu 'Tage tretende Schwäche der Contraction wird aber in dem Grade länger erhalten bleiben, als etwa von in der Wunde liegen gebliebenem Blutgerinnsel, Detritusmasse oder nachfolgender Eiterung der auf die Ganglienzelle wirkende hemmende Reiz unterhalten wird. - Ferner ist aber auch die Einwirkung eines groben mechanischen Eingriffs in das Gehirn auf die Psyche der Thiere in Betracht zu ziehen. Wahrnehmung, Vorstellung und Wille, der ganze Charakter der Thiere erscheint nach der Operation verändert. Dieselben würden möglicherweise ohne diese Allgemeinstörung den Ausfall von Innervation, der ihnen durch den Verlust von Ganglienzellengruppen erwächst, viel besser decken, und die Innervation viel besser regeln können, als es unter den vorhandenen Verbiiltnissen möglich ist. Die nach den Operationen vorhandene Erregung hindert die Thiere an einer genauen und entsprechenden Abmessung der insbesondere für die Muskelbewegung nothwendigen Impulse, dieselben fallen intensiver aus und fliessen auf den erhaltenen Leitungsbahnen, energischer als in der Norm, besonders dann wenn anderweitige äussere Reize hinzukommen. Daher die heftigen, schlecht abgemessenen Bewegungen sowohl beim Zurückziehen der in perverser Lage befindlichen Extremitäten, als auch beim Laufen. - Alle diese sofort nach der Operation zu beobachtenden Erscheinungen müssen eine allmähliche $\mathrm{Ab}$ schwächung erfahren. Zuerst muss die Wirkung der durch das Trauma gesetzten Reize auf den Charakter des Thieres erlöschen, mit ihm der Reiz auf die durch die Erregung fehlerhaft in Action gesetzten Ganglienzellengruppen. Damit versehwindet das Aussenden von ungewohnten, überreichen Impulsen auf anderen als den gewohnten Bahnen. Auch hier ist natürlich vorausgesetzt, dass nicht durch zurückbleibende Fremdkörper, Blut, Detritus, entstandene Eiterung der Reiz bis zu einem gewissen Grade erhalten bleibt. Allmählich gewinnt das Thier die Fähigkeit früher für den gleichen Zweck seltener oder ausnahmsweise, ja vielleicht noch gar nicht verwendete Ganglienzellen an Stelle der verloren gegangenen in den Gebrauch einer bestimmten beabsichtigten Leistung zu ziehen, und auch hier vorausgesetzt, dass keine neven störenden Momente hinzukommen und dass der ge- 
setzte Defect natürlicherweise nicht zu gross ist, kann eine völlige Restitution anfänglich verloren. gegangener Function Statt haben, und dies war es, was wir bei unseren sorgfältig antiseptisch operirten und gut gepflegten Thieren zu beobachten im Stande waren. -

Aus der entwickelten Vorstellung, dass die einzelnen Muskelbewegungen nicht sowohl durch die Action einzelner und gesonderter Ganglienzellen, als durch verschieden combinirte Thätigkeit grosser Gruppen zu Stande kommen, dass der Reiz durchtrennter Nervenbahnen an sich, sowie die Aenderung der Psyche der Thiere als wichtige Factoren mitwirken, erklären sich alle von uns gemachten Beobachtungen; auch sind hiernach die Beobachtungen der früher erwähnten Autoren, wenn man die von ihnen geübten abweichenden Operationsverfahren mit allen daran geknüpften Consequenzen von verschiedenen Reizwirkungen im Auge behält, wohl zu erklären. Wir können aber nach allen von uns wahrgenommenen Erscheinungen za keinem anderen Schluss kommen, als dass die Nuclei caudati keine andere Bedeutung für die von uns beobachteten Functionen haben, als die darüber liegende Hirnrinde. Dieselben bilden ein Plus oder genauer gesagt einen integrirenden Theil zu dem darüber liegenden Ganglienapparat der Rinde.

Wir haben nur eine einzige Thatsache kennen gelernt, wolche mit dieser Deutung nicht völlig vereinbar scheint, das ist die Steigerung der Körpertemperatur bei Verletzung der Nuclei caudati. Vielleicht sind die Corpora striata ihrer Lage nach mit anderen nervösen Apparaten so in Verbindung, daśs ihre Reizung Zellencomplexe in Erregung bringt, welche den besagten Einfluss auf die Körpertemperatur haben; vielleicht sind aber auch complicirtere Verhältnisse hier vorhanden, da es immer bemerkenswerth ist, dass Andeutungen von Temperatursteigerungen von uns auch bei solchen Thieren gesehen wurden, deren Wunden nur in den oberen Schichten des Hirnmantels sich befanden, ohne dass der Ventrikel eröffnet wurde, und deren völlig aseptischer Wundverlauf eine Complication mit accidenteller Fiebertemperatur ausschloss. 